\title{
Bilateral Single-Session Retrograde Intrarenal Surgery for the Treatment of Bilateral Renal Stones
}

\author{
Gokhan Atis, Hakan Koyuncu, Cenk Gurbuz, Faruk Yencilek, Ozgur Arikan, Turhan Caskurlu \\ Medeniyet University, Goztepe Training and Research Hospital, Urology (GA,CG,OA,TC) and Yeditepe \\ University Medical School, Urology (HK,FY), Istanbul, Turkey
}

\section{ABSTRACT}

Purpose: The aim of the study was to evaluate the efficacy and safety of bilateral single-session retrograde intrarenal surgery in the treatment of bilateral renal stones.

Materials and Methods: From December 2008 to February 2012, 42 patients who had undergone bilateral single-session retrograde intrarenal surgery (RIRS) and laser lithotripsy were included in the study. The procedures were performed in the lithotomy position on an endoscopy table under general anesthesia, beginning on the side in which the stone size was smaller. Plain abdominal radiography, intravenous urograms (IVU), renal ultrasonography (USG) and / or non-contrast tomography (CT) scans were conducted for all patients. The success rate was defined as patients who were stone-free or only had residual fragment less than $4 \mathrm{~mm}$.

Results: A total of 42 patients (28 male, 14 female) with a mean age $39.2 \pm 14.2$ were included in the present study. The mean stone size was $24.09 \pm 6.37 \mathrm{~mm}$ with a mean operative time of $51.08 \pm 15.22$ minutes. The stone-free rates (SFR) were $92.8 \%$ and $97.6 \%$ after the first and second procedures, respectively. The average hospital stay was $1.37 \pm 0.72$ days. In two patients (4.7\%), minor complications (Clavien I or II) were observed, whereas no major complications (Clavien III-V) or blood transfusions were noted in the studied group.

Conclusions: Bilateral single-session RIRS and laser lithotripsy can be performed safely and effectively with a high success rate and low complication rate in patients with bilateral renal stones.

\section{ARTICLE INFO}

\section{Key words:}

Kidney Calculi; General

Surgery; Renal Calculi

Int Braz J Urol. 2013; 39: 387-92

Submitted for publication:

September 09, 2012

Accepted after revision:

April 04, 2013

\section{INTRODUCTION}

Percutaneous nephrolithotomy (PCNL), shock wave lithotripsy (SWL), and retrograde intrarenal surgery (RIRS) are the most widely used treatment modalities for the management of renal stones. The 2012 European Association of Urology (EAU) guidelines on urolithiasis recommends SWL as the first treatment of choice for renal stones $<20 \mathrm{~mm}$ and PCNL for renal stones $>$ $20 \mathrm{~mm}$ located within the renal pelvis and upper or middle calices (1). Either PCNL or RIRS are re- commended for stones $>15 \mathrm{~mm}$ located within the lower pole due to the limited efficacy of SWL for stones of this size (1).

In the literature, different surgical procedures have been determined to treat bilateral renal calculi such as staged PCNL, synchronous bilateral PCNL, synchronous PCNL with contralateral ureterorenoscopy (URS), staged bilateral SWL and simultaneous SWL (2-5). Additionally, Chung et al. demonstrated the feasibility of simultaneous 
bilateral RIRS (sb-RIRS) in four patients with bilateral renal stones and significant comorbidities (6). However, the safety and efficacy of bilateral single-session RIRS has been poorly investigated. In this study, we assessed our treatment outcomes in patients undergoing bilateral single-session RIRS for bilateral renal stones.

\section{MATERIAL AND METHODS}

We performed a retrospective analysis of 42 evaluated patients with bilateral renal calculi, who underwent bilateral single-session RIRS at two referral hospitals in Turkey from December 2008 to February 2012. The selection criteria for this intervention were patients preference, failure of other treatments and multicalyceal stones. All patients were evaluated with serum biochemistry, urinanalysis, urine culture, plain radiography of kidney-ureter-bladder (KUB), IVU, renal USG and/ or CT. The stone size was determined by measuring its maximum diameter using KUB. Patients who had positive urine cultures were treated with the appropriate antibiotics before surgery. In all patients, the procedures were performed in the lithotomy position under general anesthesia, beginning on the side in which the stone size was smaller. To dilate the ureter and insert a hydrophilic guidewire to the renal pelvis, semirigid ureteroscopy was initially performed. A ureteral access sheath (UAS) was placed through the hydrophilic guidewire in all cases. The dilation of the ureteral orifice was performed using balloon dilators when the ureteroscope did not pass with ease. A flexible ureteroscope (f-URS) was placed through the UAS and the stones were fragmented using the Ho:YAG laser with a $273 \mu$ laser fiber at 0.6-1.0 J energy and 5-10 Hz frequency levels. The relocalization of the lower pole stones to the renal pelvis or upper pole was performed by basketing, when achievable, to facilitate better visualization during the lithotripsy. At the end of the procedure, a pigtail stent was placed, according to the surgeon's preference. The same procedures were then performed for the contralateral side of the renal unit that contained calculi.

At the follow-up evaluation, serum biochemistry, a post-operative plain film and renal ultrasonography were conducted at postoperative day one and repeated serum biochemistry, ultrasonography and IVU were performed at one month after the surgery to determine the presence of obstructions, clinically significant renal fragments and ureteral strictures. The success rate was defined as patients who were stone-free or only had residual fragment $<4 \mathrm{~mm}$. CT was conducted only in patients with residual stones, which were present in 3 patients (7.1\%).

Statistical analysis was performed using SPSS, version 17.0. A paired sample t-test was used to compare the pre-operative and post-operative serum creatinine levels. A p value $<0.05$ was considered statistically significant.

\section{RESULTS}

The pre-operative characteristics of the patients are summarized in Table-1. A total of 42 patients (28 male, 14 female) with a mean age $39.2 \pm 14.2$ were included in the present study. The localizations of the 134 renal stones were as follows: 51 (38.05\%) in the lower calyx of the kidney, 41 (30.59\%) in the middle calyx, 22 (16.41\%) in the renal pelvis, and 20 (14.92\%) in the upper calyx. In $12(28.5 \%)$ of the 42 patients, the following previous interventions were noted: PCNL in $4(9.5 \%)$ patients, pyelolithotomy in $3(7.1 \%)$ patients, SWL in $3(7.1 \%)$ patients and more than one of these procedures in $2(4.7 \%)$ patients.

The mean stone size was $24.09 \pm 6.37 \mathrm{~mm}$. The mean operative time was $51.08 \pm 15.22 \mathrm{mi}-$ nutes, and the mean fluoroscopy screening time was $39.26 \pm 9.87$ seconds. The SFR after the first procedure was $92.8 \%$. Three patients with residual stones underwent additonal RIRS. Two of them became stone-free, resulting in overall SFR of $97.6 \%$. Stone analyses were conducted in 30 patients (71.4\%) and the most frequent stone composition was noted as calcium oxalate.

The dilation of the ureteral orifice was performed in 26 of 84 renal units (30.95\%) and UASs were placed in all renal units. Bilateral stents were placed in 30 patients (71.4\%) and unilateral stents were placed in 12 patients $(28.6 \%)$. Stent(s) were removed approximately two weeks after the procedure. The average hospital stay was $1.37 \pm 0.72$ 
Table 1 - Patient and Stone characteristics.

\begin{tabular}{lc}
\hline Variable & Value \\
\hline Age (y) & $39.2 \pm 14.2$ \\
Gender (n) & $14(33.3 \%)$ \\
Female & $28(66.6 \%)$ \\
Male & \\
Previous renal intervention (n) & $3(7.1 \%)$ \\
SWL & $4(9.5 \%)$ \\
PCNL & $3(7.1 \%)$ \\
Open surgery & $2(4.7 \%)$ \\
More than 1 & $1.21 \pm 0.37$ \\
Pre-operative serum creatinine (mg/dL) \\
Stone size (mm) \\
Stone location (n) \\
Renal pelvis \\
Upper calyx
\end{tabular}

days. In two patients (4.7\%), minor complications (Clavien I or II) were observed, whereas no major complications (Clavien III-V) or blood transfusions were noted in the studied group (Table-2).

The mean serum creatinine levels before and after one month following the procedures (after two weeks following pigtail stent removal) were $1.21 \pm 0.37 \mathrm{mg} / \mathrm{dL}$ and $1.26 \pm 0.41 \mathrm{mg} / \mathrm{dL}$, respectively. There was no statistically significant difference between pre-operative and post-operative serum creatinine levels $(\mathrm{p}=0.89)$.

\section{DISCUSSION}

Several published articles have outlined the treatment modalities used for patients with
Table 2 - Operative and postoperative outcomes.

\begin{tabular}{lc}
\hline Variable & Value \\
\hline Mean operation time (min) & $51.08 \pm 15.22$ \\
Mean fluoroscopy time (sec) & $39.26 \pm 9.87$ \\
Stone clearance rate & \\
$\quad$ After first session & $39(92.8 \%)$ \\
$\quad$ After second session & $41(97.2 \%)$ \\
Post-operative serum creatinine (mg/dL) & $1.26 \pm 0.41$ \\
Minor complication rate & $2(4.7 \%)$ \\
Mean hospitalization time (day) & $1.37 \pm 0.72$ \\
\hline
\end{tabular}

bilateral renal stones and one of the primary surgical modalities used to treat these stones is PCNL. This procedure can be administered in either a staged, synchronous or simultaneous manner $(2,7,8)$. It has been reported that bilateral simultaneous PCNL has some unique advantages such as shorter hospital stay, less radiation exposure, reduced anesthesia and medication requirements, as well as cost-effectiveness $(2,7,9)$. However, the complication rates are similar to bilateral staged or unilateral PCNL $(2,10)$. Handa et al. examined the effects of simultaneous bilateral PCNL on bilateral renal function in pigs, and they reported that bilateral functional responses of kidneys were comparable to those observed after unilateral PCNL (11). Despite the reported efficacy and safety of bilateral PCNL procedures, some major complications, such as drops in hemoglobin that require blood transfusions and hydropneumotoraces, may still occur $(7,12)$.

SWL is another treatment modality used to manage bilateral renal calculi, which can also be applied in a simultaneous or staged manner $(4,5)$. In the study of Pienkny et al., the investigators compared the effects on renal function between simultaneous versus staged SWL, and they found no difference between the two groups (4). Perry et al. evaluated 120 patients who had undergone bilateral synchronous SWL (5). They reported a bilateral 
SFR of $60 \%$ after a single treatment without any major complications, such as renal failure or bilateral renal obstruction, however, $16 \%$ of the patients required additional procedures for residual stones.

PCNL and contralateral URS can also be used to treat bilateral urolithiasis. Mason et al. reported a efficacy rate of $92.3 \%$ and $100 \%$ after one and two sessions, respectively, in patients who underwent synchronous PCNL and contralateral URS (3). Although these complication rates were similar to those observed with unilateral PCNL in this cohort of patients, 7.7\% of them experienced major complications after this treatment modality.

The 2012 EAU urolithiasis guidelines do not recommend fURS as first-line treatment for stones $>15 \mathrm{~mm}$ in the renal pelvis and upper or middle calices, because of the decreased SFR after fURS as well as the requirement for multiple procedures to treat these stones (1). At the other site, the guidelines recommend PCNL or fURS for stones $>15 \mathrm{~mm}$ that are located in the lower pole, given that SWL has limited efficacy in the treatment of these stones (1). Despite these recommendations, there have been various published articles reporting high success rates with fURS, even for high stone burdens in any kidney location (1315). The retrograde intrarenal stone surgery is gaining more popularity day by day, given that it offers similar SFR and lower complication rates compared with PCNL, as well as and higher success rates compared with SWL (13-15).

Only a few studies have examined the safety and efficacy of RIRS in treating bilateral renal stones. In 2005, Chon et al. first reported the efficacy of SB-RIRS (16). In another study by the same investigators, they assessed their treatment outcomes in four patients with significant comorbidities who had undergone SB-RIRS, and the authors observed no major complications (6). In these studies, the procedures were performed by two surgeons who operated simultaneously. The disadvantage of this technique is the requirement of two sets of equiment such as the fURS and laser lithotriptor as well as two surgeons. In the present study, we performed single-session RIRS in 42 patients with bilateral renal calculi. Because the smaller stone size was associated with lower operative times, the procedures were initiated at the side in which the stone size was smaller. After completing one side, the RIRS was performed for the other side. We did not have to stop the procedure once the initial side was completed. On the other hand, it may be possible to end the procedure after completing one side, so the procedures can be also initiated at the symptomatic side. Our technique is advantageous because it requires only one set of equipment and a single surgeon. Furthermore, the present technique allows for the treatment of bilateral renal calculi in one anesthetic session.

Huang et al. examined bilateral RIRS in 25 patients with bilateral renal stones and reported an overall SFR of 70\%, 92\% and 92\% after first, second and third procedures, respectively (17). Similarly, the SFRs in our study group were $92.8 \%$ and 97.2\% after the first and second sessions of RIRS, respectively. Although the mean stone size was smaller than that reported in the previously published articles that have evaluated the outcomes of bilateral PCNL, we achieved a similar SFR to those studies following a bilateral PCNL. Additionally, our SFR was higher when compared with the published articles on bilateral SWL, and only $7.2 \%$ of patients in our study group required additional procedures, which is lower than the typical rates following SWL.

Bilateral single ureteroscopic procedures for ureteral calculi have been performed by several investigators. Some of these investigators have reported that bilateral calculi can be managed by bilateral URS, with low complication and high success rates $(18,19)$. In contrast, Hollenbeck et al. reported a complication rate of $26 \%$ in same session of ureteroscopic procedures (20). In their study, the post-operative complications included post-operative pain, urosepsis, urinoma and pulmonary embolus resulting in death. In the present study, only 2 patients (4.7\%) experienced minor complications. One patient had fever post-operatively and was treated with antibiotics. The other patient exhibited bleeding without the need for a transfusion. None of the patients experienced any major complications. In any of the semirigid and flexible ureteroscopic procedures, an increase in the renal pelvic pressure may occur through irrigation. The increased renal pelvic pressure can lead to infec- 
tious complications due to intrarenal, pyelovenous and pyelolymphatic backflow (21). To decrease the pelvic pressure, the use of UAS has been suggested, as well as irrigation with isoproterenol and limiting the operative time $(22,23)$. Although the UAS may fail in some circumstances such as ureteral stenosis or kinking, we were able to place the UAS in all renal units in the present study group. Our relatively low complication rate may be due to our use of UAS and our low mean operating times.

The reported mean operative times for bilateral PCNL may vary from 46 to 240 minutes (3). Additionally, Mason et al. reported an average of 149 minutes operation time for bilateral calculi that were treated with synchronous PCNL and contralateral URS (3). In the present study, the mean operative time was $51.09 \pm 15.22$ minutes which is lower than that reported for other techniques. While there is no need to reposition the patient from the supine to prone position in RIRS, it provides an advantage for minimizing operative time as compared with PCNL. At the contralateral site, the short operative time may also be due to our lower mean stone size as compared with to those in other PCNL cases.

According to the recommendations of EAU guidelines on urolithiasis, the placement of a ureteral stent after an uncomplicated URS is optional and it should be inserted in cases with a risk of postoperative complications, such as perforation, bleeding, urinary tract infection, residual fragments and pregnancy (1). After the bilateral ureteroscopic procedures, bilateral ureteral edema, leading to acute renal failure, may develop (18). Although we did not observe any intraoperative complications, at least an unilateral pigtail stent was placed to prevent acute renal failure, and none of the patients experienced such complication after the procedure. Because the operative time was too short, we placed only an unilateral stent in 12 patients. In the remaining 30 patients, bilateral pigtail stents were placed due to the above concerns.

The present study has certain limitations. First, this study was a retrospective analysis of the patients who underwent bilateral same session RIRS for bilateral renal stones, which were performed by more than one surgeon. Second, we did not evaluate the cost-analysis of the technique and post-operative pain scores and did not compare them to the other techniques that can be used to manage bilateral renal stones. Third, a post-operative plain film and renal ultrasonography were used in most of the cases to assess residual fragments, which may overestimate our SFRs post-operatively. Despite these limitations, the present study is the largest series in the literature that evaluates the outcomes of bilateral RIRS in the treatment of bilateral renal calculi.

\section{CONCLUSIONS}

Bilateral RIRS is a safe and effective treatment choice for the management of bilateral renal calculi. The procedure has a high success rate with minimal morbidity, and precludes the need for multiple procedures. Multiple studies comparing the outcomes of PCNL, SWL and RIRS are needed in this population of patients.

\section{ABBREVIATIONS}

RIRS = Retrograde intrarenal surgery

IVU = Intravenous urograms

USG = Ultrasonography

$\mathrm{CT}=$ Computed tomography

PCNL = Percutaneous nephrolithotomy

SWL $=$ Shock wave lithotripsy

EAU $=$ European Association of Urology

URS = Ureterorenoscopy

sb-RIRS = Simultaneous bilateral RIRS

KUB $=$ Kidney-ureter-bladder

UAS $=$ Ureteral access sheath

f-URS = Flexible ureteroscope

$\mathrm{SFR}=$ Stone-free rate

\section{CONFLICT OF INTEREST}

None declared.

\section{REFERENCES}

1. Türk C, Knoll T, Petrik A, Sarica K, Straub M, Seitz C: Guidelines on Urolithiasis. EAU 2012; 2-102. Available at http:// www.uroweb.org/gls/pdf/20_Urolithiasis_LR\%20March\%20 13\%202012.pdf 
2. Silverstein AD, Terranova SA, Auge BK, Weizer AZ, Delvecchio FC, Pietrow PK, et al.: Bilateral renal calculi: assessment of staged $v$ synchronous percutaneous nephrolithotomy. J Endourol. 2004; 18: 145-51.

3. Mason BM, Koi PT, Hafron J, Milhoua P, Hoenig DM: Safety and efficacy of synchronous percutaneous nephrostolithotomy and contralateral ureterorenoscopy for bilateral calculi. J Endourol. 2008; 22: 889-93.

4. Pienkny AJ, Streem SB: Simultaneous versus staged bilateral extracorporeal shock wave lithotripsy: long-term effect on renal function. J Urol. 1999; 162: 1591-3.

5. Perry KT, Smith ND, Weiser AC, User HM, Kundu SD, Nadler RB: The efficacy and safety of synchronous bilateral extracorporeal shock wave lithotripsy. J Urol. 2000; 164: 644-7.

6. Chung SY, Chon CH, Ng CS, Fuchs GJ: Simultaneous bilateral retrograde intrarenal surgery for stone disease in patients with significant comorbidities. J Endourol. 2006; 20: 761-5.

7. Bagrodia A, Raman JD, Bensalah K, Pearle MS, Lotan Y: Synchronous bilateral percutaneous nephrostolithotomy: analysis of clinical outcomes, cost and surgeon reimbursement. J Urol. 2009; 181: 149-53.

8. Ugras MY, Gedik E, Gunes A, Yanik M, Soylu A, Baydinc C: Some criteria to attempt second side safely in planned bilateral simultaneous percutaneous nephrolithotomy. Urology. 2008; 72: 996-1000.

9. Maheshwari PN, Andankar M, Hegde S, Bansal M: Bilateral single-session percutaneous nephrolithotomy: a feasible and safe treatment. J Endourol. 2000; 14: 285-7.

10. Holman E, Salah MA, Tóth C: Comparison of 150 simultaneous bilateral and 300 unilateral percutaneous nephrolithotomies. J Endourol. 2002; 16: 33-6.

11. Handa RK, Johnson CD, Connors BA, Gao S, Evan AP, Miller $N L$, et al.: Renal functional effects of simultaneous bilateral single-tract percutaneous access in pigs. BJU Int. 2010; 105: 125-8.

12. Shah HN, Kausik VB, Hegde SS, Shah JN, Bansal MB: Safety and efficacy of bilateral simultaneous tubeless percutaneous nephrolithotomy. Urology. 2005; 66: 500-4.
13. Wiesenthal JD, Ghiculete D, D'A Honey RJ, Pace KT: A comparison of treatment modalities for renal calculi between 100 and $300 \mathrm{~mm} 2$ : are shockwave lithotripsy, ureteroscopy, and percutaneous nephrolithotomy equivalent? J Endourol. 2011; 25: 481-5.

14. Bozkurt OF, Resorlu B, Yildiz Y, Can CE, Unsal A: Retrograde intrarenal surgery versus percutaneous nephrolithotomy in the management of lower-pole renal stones with a diameter of 15 to $20 \mathrm{~mm}$. J Endourol. 2011; 25: 1131-5.

15. Breda A, Ogunyemi 0, Leppert JT, Schulam PG: Flexible ureteroscopy and laser lithotripsy for multiple unilateral intrarenal stones. Eur Urol. 2009; 55: 1190-6.

16. Chon $\mathrm{CH}$, Chung SY, Ng CS, Fuchs GJ: Simultaneous bilateral retrograde intrarenal surgery for bilateral complex upper tract stone disease. Urology. 2005; 65: 572-4.

17. Isen K: Single-session ureteroscopic pneumatic lithotripsy for the management of bilateral ureteric stones. Int Braz J Urol. 2012; 38: 63-8.

18. Huang Z, Fu F, Zhong Z, Zhang L, Xu R, Zhao X: Flexible ureteroscopy and laser lithotripsy for bilateral multiple intrarenal stones: is this a valuable choice? Urology. 2012; 80: 800-4.

19. Gunlusoy B, Degirmenci T, Arslan M, Kozacioğlu Z, Nergiz N, Minareci S, et al.: Bilateral single-session ureteroscopy with pneumatic lithotripsy for bilateral ureter stones: feasible and safe. Urol Int. 2008; 81: 202-5.

20. Hollenbeck BK, Schuster TG, Faerber GJ, Wolf JS Jr.: Safety and efficacy of same-session bilateral ureteroscopy. J Endourol. 2003; 17: 881-5.

21. Binbay M, Yuruk E, Akman T, Ozgor F, Seyrek M, Ozkuvanci $\mathrm{U}$, et al.: Is there a difference in outcomes between digital and fiberoptic flexible ureterorenoscopy procedures? J Endourol. 2010; 24: 1929-34.

22. Jung H, Nørby B, Frimodt-Møller PC, Osther PJ: Endoluminal isoproterenol irrigation decreases renal pelvic pressure during flexible ureterorenoscopy: a clinical randomized, controlled study. Eur Urol. 2008; 54: 1404-13.

23. Auge BK, Pietrow PK, Lallas CD, Raj GV, Santa-Cruz RW, Preminger GM: Ureteral access sheath provides protection against elevated renal pressures during routine flexible ureteroscopic stone manipulation. J Endourol. 2004; 18: 33-6.

\section{Correspondence address:} Dr. Gokhan Atis

Tahrali Sitesi Şafakyeli Apt. D 36 Atasehir, Istanbul, Turkey Fax: + 9021 6521-8608

E-mail: gokhanatis@hotmail.com 